\title{
Habilidades técnicas del portero de hockey patines en la falta directa The Technical Skills of Rink Hockey Goalkeeper in Direct Free Hit
}

\author{
*Guillem Trabal Tañá, **Gabriel Daza, **Joan Riera
}

*Hockey Valdagno 1938 (Italia), **Universidad de Barcelona (España)

\begin{abstract}
Resumen. El objetivo del presente estudio fue identificar las habilidades técnicas del portero de hockey patines en las faltas directas de la alta competición, proponiendo, al mismo tiempo, la estructura funcional de las habilidades técnicas y destacando los encadenamientos más eficaces. Se ha utilizado la metodología observacional analizando las faltas directas en la liga profesional española $(n=637)$. Se creó un instrumento de observación ad hoc con las habilidades técnicas del portero, del jugador y la lateralidad del jugador. Se validó el instrumento de observación mediante el criterio de autoridad y se calculó la fiabilidad intra e inter observador. Se realizó un análisis correlacional mediante la prueba de chi cuadrado de Pearson para identificar las relaciones entre habilidades. Los resultados constatan la existencia de una relación significativa entre ambas habilidades $(\div 2=273,980$, $p<.05$ ), siendo las relaciones más estables la pantalla con los disparos y el paso de valla con los driblings, aunque esta última habilidad técnica del portero más utilizada no se presenta como la más eficaz. Se observa que los porteros responden con habilidades diferentes en función de la lateralidad del jugador. Esta información puede ayudar a los entrenadores a crear situaciones específicas de entrenamiento para mejorar el rendimiento de los porteros en las faltas directas y aportar un conocimiento específico para el portero para preparar la estrategia adecuada en las faltas directas.
\end{abstract}

Palabras clave: interacción, rendimiento, estrategia, eficacia, competición.

Abstract. The aim of the present study was to identify the technical skills of the rink hockey goalkeeper in the direct free hits in the high competition, proposing, at the same time, the functional structure of the technical skills and highlighting the most effective chains. The observational methodology was used analyzing the direct free hits in the Spanish professional league $(n=637)$. An ad hoc observation instrument was created with the technical skills of the goalkeeper, the technical skills of player and the laterality of the player. The observation instrument was validated by the authority criterion and intra and inter observer reliability was calculated. Correlation analysis was performed using Pearson's chi-square test to identify the relationships between skills. The results confirm the existence of a significant relationship between both skills $(\div 2=273.980, p<.05)$, the most stable relationships being the screen with the shots and the fence step with the driblings, although, his last technical skill of the most used goalkeeper is not the most effective. It is observed that the goalkeepers respond with different skills depending on the player's laterality. This information can help the coaches to create specific training situations to improve the performance of the goalkeepers in the direct free hits and provide a specific knowledge for the goalkeeper to prepare the appropriate strategy in the direct free hits..

Key words: interaction, performance, strategy, efficiency, competition.

\section{Introducción}

Desde la temporada 2009-10 en la que entraron en vigor nuevas reglas de juego en el hockey patines (Rink Hockey Committee, 2009), las faltas directas (FD) se han convertido en protagonistas del juego debido al aumento de la frecuencia, especialmente, en la fase final del partido y al incremento de la eficacia en los goles conseguidos mediante FD (Trabal, 2017). Desde que se han producido estos cambios, ArboixAlió \& Aguilera-Castells (2018) y Trabal (2017) han analizado la influencia de variables de juego en el rendimiento final en el hockey patines. Recientes investigaciones en balonmano (Jimenez-Olmedo, Agulló \& López, 2017; Saavedra, Porgeirsson, Kristjánsdóttir, Chang \& Halldórsson, 2017) y en waterpolo (Pérez, Ordóñez \& González, 2018) sitúan la actuación del portero como una variable que condiciona el resultado final.

En el lanzamiento de FD se crea una relación de oposición entre el portero y el jugador en la que se genera una influencia recíproca. Entre ellos dos se establece un duelo asimétrico con roles opuestos (Parlebas, 2001): el jugador con el objetivo de marcar gol y el portero de evitarlo. El éxito de esta interacción está determinado por la pelota. En principio, la iniciativa en la acción está determinada por la posesión de la pelota, siendo en este caso concreto el jugador quien tiene el control, quien lleva la iniciativa en la interacción. Sin embargo, el portero también puede intentar llevar la iniciativa en el transcurso de esta interacción utilizando fintas o desplazándose hacia la pelota (Trabal, 2012). Esta situación de uno contra el portero se realiza sin la presencia de compañeros y adversarios. Se inicia cuando el jugador, con la pelota situada a 7,40 metros de distancia de la portería, contacta la pelota con el stick. Es en este momento cuando el portero, que se espera con posición de cuclillas situando los frenos de los patines o las ruedas delanteras sobre la línea de gol, puede moverse y realizar los movimientos que crea más adecuados (Rink Hockey Committee, 2009).

Fecha recepción: 31-07-18. Fecha de aceptación: 09-01-19 Gabriel Daza

gabidaza@gmail.com
Las intenciones tácticas se han utilizado para analizar las interacciones entre el portero y el jugador en la FD (Bayer, 1987; Espar, 2011), este planteamiento permite establecer los objetivos que determinan y jerarquizan las habilidades técnicas de los jugadores. Reconocer las intenciones tácticas permite catalogar las diferentes posibilidades de interacción del jugador. Las intenciones tácticas se manifiestan mediante las habilidades técnicas y a cada intención se le asocian una o varias habilidades. En la interacción portero contra jugador en la FD, el portero y el jugador disponen de varias posibilidades para alcanzar su objetivo, el portero para evitar el gol y el jugador para conseguirlo.

El reglamento permite al lanzador dos formas de ejecución: lanzar directamente desde el punto de FD o progresar hacia portería (Pauls, 2012). La actuación del portero, en cada uno de los casos, está muy condicionada por la decisión del lanzador (Trabal, 2012). Los dos escenarios ofrecen diferentes tipos de habilidades para ser eficaces.

Cuando el jugador lanza directo a portería, la velocidad del lanzamiento puede llegar a ser $115 \mathrm{~km} / \mathrm{h}$ si el jugador está en movimiento, y de 102 km/h si éste está parado (Vaz, Ramos, Abrantes, Queirós de Melo \& Conceição, 2011). Teniendo en cuenta que la distancia del punto de lanzamiento de la FD hasta la línea de gol es de 7,4 metros, el tiempo que tarda la pelota en llegar a la línea de portería puedellegar a ser de 0,23 segundos. Estudios en porteros de balonmano han mostrado que el tiempo de reacción de éstos oscila entre los 0,39 segundos y los 0,57 segundos (Zeier, 1987) y entre 0,63 y 0,69 segundos según si se suman los parámetros de tiempo de reacción y tiempo de movimiento (Pokrajac, 1980). Por lo tanto, ante lanzamientos directos a portería, el portero no dispone del tiempo de reacción y de movimiento suficiente, fisiológicamente no puede atender a esta demanda. Por este motivo, la intención del portero es salir frontalmente de la portería para reducir el ángulo de lanzamiento y realizar una habilidad técnica que ocupe el máximo espacio generando mucho volumen con el cuerpo, siendo la más recomendable la pantalla (PNTLL), técnica que consiste en colocar las dos piernas laterales y paralelas, al mismo tiempo que el cuerpo se sustenta sobre el guante del stick (Folguera, 2000; González, 2011; Trabal, 2012). Otra alternativa del portero es anticipar la dirección del lanzamiento y desplazar su cuerpo, o algún segmento corporal, hacia 
una zona predeterminada. Este tipo de parada se utiliza más habitualmente cuando el portero está apercibido con la sanción de tarjeta azul (Trabal, 2012).

Ante situaciones de FD que el jugador progresa hacia portería puede combinar múltiples habilidades técnicas en su ejecución (Brazio, 2006; Duque, 2004; Ferreira, 2003; Pauls, 2012). El portero debe procurar situarse lo más avanzado posible de la portería y utilizar una posición inicial para reducir el ángulo de lanzamiento del jugador para reaccionar si el jugador decide arrastrar o escupir la pelota. Al mismo tiempo, esta posición inicial debe permitir reaccionar al dribling del jugador si éste no decide arrastrar o escupir la pelota. Las dos posiciones iniciales más utilizadas por los porteros son la rodilla en el suelo y la media pantalla (Folguera, 2000; González, 2008), siendo esta última una posición inicial muy exclusiva del portero español (Trabal, 2012).

Las intenciones tácticas del portero están relacionadas con las intenciones tácticas del jugador ya que su objetivo es adaptarse de la forma más eficiente a la habilidad técnica del jugador (figura 1). A partir de las intenciones tácticas del jugador se establece una relación entre portero y jugador de reciprocidad mutua que queda manifestada por las habilidades técnicas realizadas por ambos.

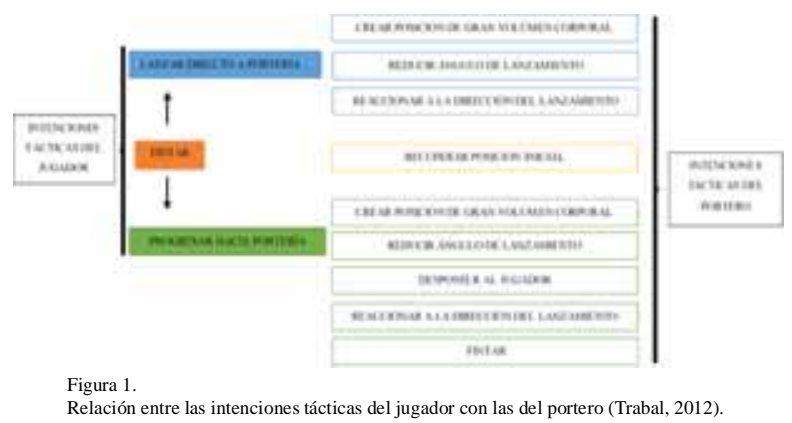

Un elemento importante a destacar en esta interacción es la lateralidad del jugador. Otros deportes ya han evidenciado la mayor dificultad de los porteros en interactuar ante los jugadores en función de la lateralidad (Hagemann, 2009), mostrando en estas interacciones una eficacia inferior de los porteros para defender lanzamientos de jugadores zurdos (Bauman, Friehe \& Wedow, 2011) y una mayor dificultad a la hora de predecir la dirección de sus lanzamientos (McMorris \& Colenso, 1996). En relación a la lateralidad de los porteros de hockey patines hay que destacar que sus movimientos son asimétricos, la obligatoriedad de coger el stick con una mano condiciona sus acciones, de esta forma, un mismo lanzamiento dirigido a la derecha o a la izquierda de portero tendrá como respuesta una habilidad técnica diferente (Trabal, 2012).

A través de la FD se puede estudiar la interacción portero-jugador en su esencia, esta interacción se presenta en un tiempo limitado, sin la presencia de compañeros ni de adversarios y esta se desarrolla de forma completa. La FD es la unidad mínima donde se manifiesta la interacción portero - jugador de forma natural y total. Dada la significación, especificidad y naturaleza de las jugadas de la FD, y en relación con la gran importancia que porteros y jugadores otorgan a estas acciones, y a la gran influencia que se otorga al portero como elemento determinante en el rendimiento de los equipos (Trabal, 2016), el objetivo del presente estudio es identificar las habilidades técnicas del portero de hockey patines en las faltas directas en la alta competición, proponiendo, al mismo tiempo, la estructura funcional de las habilidades técnicas y destacando los encadenamientos más eficaces. A través de esta investigación se pretende obtener información que ayude tanto a portero como jugador a mejorar sus habilidades en su relación uno contra uno ya que no existe ningún estudio que haya analizado ni las FD ni ningún otro tipo de interacción portero- jugador en el hockey patines.

\section{Metodología}

\section{Diseño metodológico}

Se ha utilizado la metodología observacional por las posibilidades que ofrece para analizar la interacción portero-jugador en su contexto natural (Anguera, Blanco, Losada \& Hernández-Mendo, 2000; Lasierra \& Escudero, 1993). El diseño ha sido puntual, nomotético y multidimensional. Para el estudio se han utilizado los datos de la OK LIGA, competición oficial de difusión pública, aplicando los requisitos éticos establecidos por laAmerican Psychological Association (American Psychological Association, 2002).

\section{Participantes}

La muestra ha sido formada por todas las FD lanzadas en la OK LIGA en la temporada 2015-16. En la OK 2015-16 han participado 16 equipos, se ha disputado en el transcurso de 30 jornadas, divididas en dos vueltas y con ocho partidos en cada jornada. El total de la muestra ha sido de 240 partidos en los que se han lanzado 650 FD. Finalmente, se han analizado 637 FD por la falta de calidad en la imagen de 13 FD.

\section{Instrumentos}

Se ha creado un instrumento de observación ad hoc formado por un sistema de categorías y un formato de campo. El instrumento estaba formado por cuatro criterios y 19 categorías (tabla 1). La lateralidad de los porteros no es una variable debido a que todos los porteros en la OK LIGA son diestros. Se ha validado el instrumento a través del criterio de autoridad y se han realizado las pruebas de concordancia intraobservador $(\mathrm{k}=, 992)$ y interobservador $(\mathrm{k}=, 984)$ para asegurar que el instrumento era fiable.

\begin{tabular}{|c|c|}
\hline Criterio & Categoría \\
\hline \multirow{6}{*}{ Habilidad técnica del portero (HTP) } & Pantalla (PNTLL) \\
\hline & Abertura de piernas (ABP) \\
\hline & Movimiento de brazos (MBR) \\
\hline & Estirada al guante del stick (EGS) \\
\hline & Otras habilidades técnicas (OTR) \\
\hline & Paso de valla (PASV) \\
\hline \multirow[t]{9}{*}{ Habilidad técnica del jugador (HTCJ) } & Chutar \\
\hline & Arrastrar \\
\hline & Driblar: Gancho a la cuchara (GAC) \\
\hline & Driblar: Gancho a la pala (GAP) \\
\hline & $\begin{array}{l}\text { Driblar: Gancho a la cuchara y contra gancho a la } \\
\text { pala (GCCP) }\end{array}$ \\
\hline & $\begin{array}{l}\text { Driblar: Gancho a la pala y contra gancho a la } \\
\text { cuchara (GPCC) }\end{array}$ \\
\hline & Driblar: Levantada y golpeo de pala (LGP) \\
\hline & Driblar: Levantada y golpeo de cuchara (LGC) \\
\hline & Driblar: Otros driblings (OTR) \\
\hline \multirow[t]{3}{*}{ Lateralidad del jugador } & Diestro \\
\hline & Zurdo \\
\hline & Gol \\
\hline
\end{tabular}

\section{Procedimiento}

Se han descargado los vídeos de los partidos procedentes de la plataforma de la Real Federación Española de Patinajey se han visualizado las FD a través del programa Kinovea v. 0.8.17. Se ha utilizado una plantilla de Excel como instrumento de registro. Finalmente, los datos se han volcado en el programa SPSS v.23 para realizar el análisis estadístico. Se ha realizado un análisis descriptivo de las diferentes variables: frecuencias, porcentajes de utilización y porcentajes de eficacia del portero, y un análisis correlacional mediante chi cuadrado de Pearson entre las HTCJ y HTCP para comprobar la existencia de relaciones significativas y determinar la influencia entre ambas habilidades.

\section{Resultados}

La eficacia en la marcación de FD en la OK LIGA 2015-16 es del 29,7\%, de 637 FD analizadas se han marcado 189 goles, con una correspondencia en eficacia del portero (\% EP) del 70,3\%. Se observa la existencia de relación significativa entre las HTCJ y las HTCP $(\div 2=$ 273.980, Sig. $=.000, p<.05)$.

La PNTLLy el PASV son las dos habilidades técnicas más utilizadas por los porteros. El PASV la habilidad más utilizada, 227 veces, representando el 35,6\%. La PNTLL ha aparecido en 157 FD, correspondiendo al 24,6\%. La tercera habilidad técnica más utilizada por los porteros ha sido la EGS, en $93 \mathrm{FD}$ (14,6\%).

Un análisis más detallado atendiendo a la vinculación entre las habilidades técnicas jugador y portero, permite identificar que la habili- 
dad técnica más utilizada por el portero ante los chutes es la PNTLL con un $74,5 \%$. Utilizando la PNTLL, el portero obtiene un \%EP del $76,8 \%$, siendo el porcentaje más alto conseguido por el portero ante este tipo de lanzamientos (tabla 2).

Tabla 2 .

Relación entre HTCP y HTCJ con los porcentajes de utilización y los \%EP de las habilidades más utilizadas y con mayor eficacia.

\begin{tabular}{|c|c|c|c|c|c|c|c|c|}
\hline \multicolumn{2}{|l|}{ HTCJ } & \multicolumn{3}{|c|}{ HTCP más utilizada } & \multicolumn{3}{|c|}{ HTCP con mayor \%EP } & \multirow{2}{*}{$\begin{array}{c}\text { Media del } \\
\text { \%EP ante la } \\
\text { HTCJ }\end{array}$} \\
\hline HTCJ & $\begin{array}{c}\% \\
\text { de uso }\end{array}$ & HTCP & $\begin{array}{c}\% \\
\text { de uso }\end{array}$ & $\%$ ЕP & HTCP & $\begin{array}{c}\% \\
\text { de uso }\end{array}$ & \%ЕP & \\
\hline Chutar & 17,3 & PNTLL & 74,5 & 76,8 & PNTLL & 74,5 & 76,8 & 72,7 \\
\hline Arrastrar & 8,8 & MBR & 26,8 & 86,7 & MBR & 26,8 & 86,7 & 69,6 \\
\hline Driblar & 73,9 & PASV & 45,4 & 68,2 & MBR & 7,4 & 82,9 & 69,9 \\
\hline No levantar y golpear & 81,1 & \begin{tabular}{|l|} 
PASV \\
\end{tabular} & 47,6 & 68,7 & MBR & 6,5 & 84 & 69,1 \\
\hline Levantar y golpear & 18,9 & PASV & 36 & 65,6 & PNTLL & 20,2 & 88,9 & 73 \\
\hline
\end{tabular}

Para contrarrestar la habilidad técnica del lanzador de arrastrar o escupir la pelota, el portero utiliza prioritariamente el MBR en el 26,8\% de ocasiones. El \%EP del portero conseguido utilizando esta técnica es del 86,7\%, siendo el \%EP más alto ante este tipo de lanzamientos.

Ante la HTCJ de driblar el PASV es la habilidad más utilizada por los porteros con un 45,4\% de utilización. Los \%EP ante las acciones de dribling utilizando el PASV es del 68,2\%. A pesar de esta alta utilización de la PNTLL, el portero logra un \%EP superior si utiliza el MBR,ABP o PNTLL. Un análisis más detallado de los driblings diferencia las HTCJ que finalizan con levantada y golpeo de pelota y las que no. El PASV sigue siendo el HTCP más empleada, en un $47,6 \%$, cuando no hay levantada y golpeo y en un 36\% cuando hay levantada y golpeo, siendo el \%EP en cada una de estas acciones utilizando el PASV del $65,3 \%$ de y del $68,7 \%$ respectivamente. Como se observa en la tabla 2 , en ambos casos hay otras HTCP más eficaces ante los driblings en función de si se levanta o no la pelota.

La relación entre las HTCJ y las HTCP diferenciando entre jugadores diestros y zurdos presenta resultados diferenciados en función de la lateralidad (tabla 3). En primer lugar, cabe destacar que de las 637 FD lanzadas en la OK LIGA 2015-16, 430 han sido lanzadas por jugadores diestros (67,5\%) y 207 para jugadores zurdos (32,5\%). Existe relación significativa entre las habilidades técnicas del portero y del jugador en los jugadores diestros $(\div 2=207.217$, Sig. $=, 039, p<.05)$ y en los zurdos $(\div 2=77,307$, Sig. $=.000, p<.05)$. Frente a los disparos de los diestros y de los zurdos la PNTLL es la opción más frecuente, un $73,8 \%$ en los diestros y un 76,7\% en los zurdos. El \%EP utilizando esta habilidad técnica es 74,6\% en los diestros y del 82,6\% de en los zurdos, y es con esta habilidad técnica que el portero obtiene el \%EP más alto frente a los chutes.

Tabla 3.

Relación entre HTCP y HTCJ diestros y zurdos, con los porcentajes de utilización y los \%EP de las habilidades más utilizadas y con mayor eficacia.

\begin{tabular}{|c|c|c|c|c|c|c|c|c|c|}
\hline \multirow[t]{2}{*}{ Lateralidad } & \multicolumn{2}{|c|}{ HTCJ } & \multicolumn{3}{|c|}{ HTCP más utilizada } & \multicolumn{3}{|c|}{ HTCP con mayor \%EP } & \multirow[t]{2}{*}{\begin{tabular}{|c|} 
Media del \\
\%EP ante \\
la HTCJ
\end{tabular}} \\
\hline & HTCJ & $\begin{array}{c}\% \\
\text { de uso }\end{array}$ & HTCP & $\begin{array}{c}\% \\
\text { de uso }\end{array}$ & \%ЕР & НTCP & $\begin{array}{c}\% \\
\text { de uso }\end{array}$ & \%EP & \\
\hline \multirow[t]{3}{*}{ Diestro } & Chutar & 18,6 & PNTLL & 73,8 & 74,6 & PNTLL & 73,8 & 74,6 & 73,8 \\
\hline & Arrastrar & 10,7 & MBR & 37,1 & 86,7 & MBR & 37,1 & 86,7 & 76,1 \\
\hline & Driblar & 70,7 & PASV & 49,3 & 70,7 & MBR & 7,6 & 82,6 & 71,1 \\
\hline \multirow[t]{3}{*}{ Zurdo } & Chutar & 14,5 & PNTLL & 76,7 & 82,6 & \begin{tabular}{|l|} 
PNTLL \\
\end{tabular} & 76,7 & 82,6 & 70 \\
\hline & Arrastrar & 4,8 & PNTLL & 30 & 66,7 & PNTLL & 30 & 66,7 & 40 \\
\hline & Driblar & 80,7 & PASV & 38,3 & 62,5 & PNTLL & 11,4 & 89,5 & 67,7 \\
\hline
\end{tabular}
brazos, PASV=paso de valla.

Para contrarrestar la habilidad técnica de arrastrar o escupir la pelota ante los diestros el portero utiliza prioritariamente el MBR en el 37,1\% de FD. Con esta habilidad presenta el \%EP más alto ante estos lanzamientos, del 86,7\%. Por el contrario, ante los lanzamientos de arrastre de los zurdos el portero no realiza esta habilidad y en el $30 \%$ de ocasiones utiliza una PNTLL y en otro 30\% el PASV. Con la PNTLL alcanza el \%EP más alto frente a los zurdos en los arrastres, del 50\%. Cabe destacar que de las 56 FD lanzadas con arrastre el 17,8\% han sido lanzadas por zurdos.

Ante los driblings de los diestros la habilidad técnica más empleada por los porteros es el PASV en el 49,3\% de ocasiones, ante los driblings de los zurdos también es el PASV la habilidad más recurrente en un 38,3\%. Con el PASV ante los diestros los porteros alcanzan un \%EPdel 70,7\%, superado por el \%EP cuando se realizan otras HTCP, un 82,6\% con el MBR y un 75,7\% con las EGS. El \%EP ante los driblings de los jugadores zurdos es del $67,7 \%$, una eficacia inferior a la que los porteros obtienen ante los driblings de los diestros, 71,1\%. Los \%EP más altos en la interacción de dribling con los zurdos se obtienen con la PNTLL (89,5\%) y con el MBR (83,3\%), los \%EP más bajos son con el PASV (62,5\%) y con la EGS $(56,8 \%)$.

Para un mejor conocimiento de la relación entre las habilidades técnicas del portero y del jugador se ha profundizado en el análisis del dribling que es el HTCJ más utilizada en las FD (73,9\%). Dentro de esta categoría de dribling hay dos habilidades técnicas que son las más utilizadas por los jugadores: el gancho a la cuchara (GAC) y el gancho a la cuchara y contra gancho a la pala (GCCP). En este caso, el análisis se ha realizado diferenciando los jugadores en función de la lateralidad. Ante el GAC de los jugadores diestros los porteros utilizan con más frecuencia el PASV (55,8\%), alcanzando un \%EP el 74,8\%, a pesar de que el MBR es la HTCP con un \%EP más alto (83,3\%). En el GAC de los zurdos los porteros mayoritariamente responden con una estirada al guante del stick(EGS) (65,5\%). Con esta habilidad técnica el\%EP es del $52 \%$, una eficacia superada cuando se utiliza el PASV (87,5\%). Cuando los jugadores realizan el GCCP el portero tiene preferencia para responder con el PASV, ya sea ante los jugadores diestros (61,3\%) como ante los zurdos (37,9\%). A pesar de estas preferencias, el portero logra \%EP superiores cuando utiliza otras habilidades técnicas, ante los diestros con la utilización de laEGS obtiene un \%EP del 87,5\% y ante los zurdos la abertura de piernas (ABP) permite llegar hasta al 88,9\% (tabla 4).

Tabla 4.

Relación entre las habilidades de dribling del jugador: GAC y GCCP con los porcentajes de utilización y los \%EP de las habilidades más utilizadas y con mayor eficacia.

\begin{tabular}{|c|c|c|c|c|c|c|c|c|c|}
\hline \multirow[t]{2}{*}{ Lateralidad } & \multicolumn{2}{|c|}{ HTCJ } & \multicolumn{3}{|c|}{ HTCP más utilizada } & \multicolumn{3}{|c|}{ HTCP con mayor \%EP } & \multirow[t]{2}{*}{$\begin{array}{c}\text { Media del } \\
\text { \%EP ente la } \\
\text { HTCJ } \\
\end{array}$} \\
\hline & & $\begin{array}{c}\% \\
\text { de uso }\end{array}$ & НTCP & $\begin{array}{c}\% \\
\text { de uso }\end{array}$ & \%EP & НTCP & $\begin{array}{c}\% \\
\text { de uso }\end{array}$ & $\%$ EP & \\
\hline \multirow[t]{2}{*}{ Diestro } & GAC & 27,9 & PASV & 55,8 & 74,6 & MBR & 5 & 83,3 & 70 \\
\hline & GCCP & 14,3 & PASV & 61,3 & 53,3 & EGS & 25,8 & 87,5 & 72,6 \\
\hline \multirow[t]{2}{*}{ Zurdo } & GAC & 19,3 & EGS & 65,5 & 52 & PASV & 20 & 87,5 & 65 \\
\hline & GCCP & 14,4 & PASV & 37,9 & 63,6 & $\mathrm{ABP}$ & 31 & 88,9 & 72,4 \\
\hline
\end{tabular}

Leyenda: HTCJ=Habilidad técnica del jugador, HTCP=Habilidad técnica del portero, $\% \mathrm{EP}=$ porcentaje eficacia del portero, \%=porcentaje, PNTLL=pantalla, MBR=movimiento de brazos, $\mathrm{PASV}=$ paso de valla, EGS=estirada al guante del stick, $\mathrm{ABP}=$ abertura de piernas, $\mathrm{GAC}=$ gancho a la cuchara, GCCP=gancho a la cuchara y contra gancho a la pala.

\section{Discusión}

El objetivo del presente estudio ha sido identificar las habilidades técnicas del portero de hockey patines en las faltas directas en la alta competición, proponiendo, al mismo tiempo, la estructura funcional de las habilidades técnicas y destacando los encadenamientos más eficaces.

La relación entre las HTCJ y las HTCP se explican por la relación de interacción con influencia recíproca creada entre portero y jugador en la acción de uno contra el portero en la FD. Ante las acciones de chute los porteros tienen una clara preferencia por la utilización de la PNTLL. Esta habilidad técnica es elegida por el portero con la voluntad de crear una posición con el cuerpo que ocupe el máximo de volumen posible (Folguera, 2000; Trabal, 2012). Ante este tipo de lanzamientos, en los que la velocidad de la pelota llega a ser de más de $100 \mathrm{~km} / \mathrm{h}$ (Vaz et al., 2011), lo más recomendable es realizar una habilidad técnica con el cuerpo que permita ocupar el máximo de espacio posible y reducir el ángulo de lanzamiento, pues el portero no puede esperar a intentar detectar la trayectoria del lanzamiento y realizar una habilidad técnica después de detectar esta dirección (Trabal, 2012). El hecho de que con la PNTLL el portero alcance los \%EP más altos ante los disparos confirma las recomendaciones de Folguera (2000) y Trabal (2012) de utilizar la PNTLL ante los disparos.

Ante los arrastres la respuesta más utilizada por los porteros son los MBR (26,8\%), estas habilidades se consideran las más adecuadas porque la velocidad de este tipo de lanzamientos es más baja que la de los disparos y eso da más tiempo de reacción al portero. Desde la 
posición inicial que adopta el portero los movimientos de los brazos permiten reaccionar a la trayectoria de la pelota y desplazar los brazos para contactar con esta (Trabal, 2012). En este caso, los MBR han demostrado ser la habilidad técnica adecuada ante los lanzamientos de arrastre, el portero consigue detener el 86,7\% de las FD, unos valores muy superiores a la media de \%EP ante los arrastres que es del 69,6\% y confirma las recomendaciones de Trabal (2012) en utilizar esta habilidad ante este tipo de lanzamientos.

Cuando el jugador decide avanzar hacia portería y driblar al portero, este tiene una alta preferencia por el PASV (45,4\%). El PASV está fuertemente relacionado con la posición inicial que adopta el portero previamente a realizar una habilidad técnica para detener el dribling. El portero de hockey patines tiene la tendencia de posicionarse con la rodilla en el suelo en las situaciones que el jugador avanza a portería para driblar, en el 59\% de FD que el jugador dribla al portero se coloca en esta posición inicial (Trabal, 2017). Desde la rodilla en el suelo realizar un PASV es la habilidad técnica más cómoda de realizar ya que el portero únicamente debe dejar caer la zona glútea en el suelo y estirar la pierna. Es importante destacar que, ante los driblings, el portero no utiliza la habilidad técnica que permite alcanzar los \%EP más altos, esto demuestra que el portero utiliza el PASV porque es la opción más cómoda y rápida a partir de la posición inicial de la rodilla en el suelo a pesar de que esta habilidad no es la que permite conseguir mayor eficacia. En referencia al \%EP se observa que cuando el portero utiliza el MBR logra la eficacia más alta. El portero realiza estos movimientos con los brazos para detener el lanzamiento cuando el dribling del jugador es de amplitud corta y no exige al portero de tener que realizar ninguna otra habilidad de más amplitud para detener el lanzamiento y simplemente, a partir de la posición inicial del portero, este realiza un movimiento con los brazos para detener el lanzamiento. Por tanto, en este caso, la responsabilidad de que el \%EP más alto ante los driblings sea alcanzado con el MBR se explica por las habilidades técnicas del jugador de menor calidad y corta amplitud.

La relación de los porteros con los jugadores en las acciones de dribling más utilizadas, GAC y GCCP, refleja claramente una diferencia de la respuesta del portero en función de la lateralidad del jugador. Aunque jugadores diestros y zurdos utilizan las mismas habilidades técnicas, estas tienen una influencia diferente en el portero debido a que las HTCP son asimétricas, la obligatoriedad de coger el stick con un guante condiciona todos los movimientos a realizar por el portero. De esta forma, una misma habilidad técnica realizada por jugadores zurdos o diestros es manifestada e influye de diferente forma ante el portero. En el caso concreto del GAC de un jugador diestro este finaliza al lado izquierdo del portero, donde en la mayoría de habilidades técnicas está el guante libre y la pierna del guante libre, por lo que la respuesta del portero en PASV es un movimiento del todo natural ante estas acciones. Por el contrario, el mismo GAC realizado por un zurdo finaliza a la derecha del portero, donde generalmente este tiene el guante del stick, el casco y el pecho. En este caso, responder con una EGS tiene sentido porque es una HBTP que al portero le permite transportar rápidamente la cabeza, el pecho y el guante del stick al lado donde se ha desplazado la pelota. La utilización de estas HBTP queda justificada por la búsqueda de la facilidad y de la comodidad en la respuesta por parte del portero ante las HTCJ. Sin embargo, es importante destacar que ante el GCCP de diestros y zurdos el portero tiene un \%EP muy bajo utilizando el PASV y la EGS, este hecho demuestra que por mucho que el portero utilice las habilidades que son más cómodas de realizar ante las acciones del jugador, en este caso el PASV, estas no son las más recomendadas. Lo mismo sucede cuando los jugadores zurdos realizan el GAC, el portero responde con un EGS por comodidad a pesar de que el PASV es más adecuado. Un dato que ayuda a comprender la importancia que tiene en el hockey patines las asimetrías observadas en los porteros en función de la lateralidad de los jugadores se presenta en los disparos. Ante estas acciones en las que no existe desplazamiento del stick y la pelota por parte del jugador, debido a que se realiza un lanzamiento directo, el portero tiene una respuesta y una eficacia similar utilizando la PNTLL tanto ante diestros como ante zurdos.
La principal limitación del estudio ha sido la falta de estudios previos sobre la FD y sobre el portero de HP y el tamaño y la naturaleza de la muestra.

\section{Conclusiones}

A través de la FD se ha podido analizar la interacción portero jugador debido a que esta acción es la unidad mínima donde se manifiesta la interacción portero - jugador de forma natural y total. Las habilidades técnicas más utilizadas por el portero son el PASV $(35,6 \%)$ y la PNTLL (24,6\%). Entre portero y jugador se establecen relaciones estables en la utilización de las habilidades técnicas de ambos. Este hecho confirma que las FD son situaciones portero contra jugador en las que existe influencia recíproca. Las dos relaciones más estables son la utilización de la PNTLL ante los disparos (74,5\%) y el PASV ante los driblings (45,4\%). Las relaciones establecidas por el portero no demuestran ser siempre las más eficaces, aunque ante los chutes y arrastradas el portero utiliza de forma más frecuente la HTCP con la que obtiene el \%EP más alta, del 76,8\% y del 86,7\% respectivamente, la utilización preferencial del PASV en los driblings está condicionada por la comodidad del portero y no tanto por su efectividad ( $\% \mathrm{EP}=68,2 \%$ ).

El conocimiento adquirido en esta investigación permite conocer cuáles son las habilidades técnicas del portero más adecuadas para responder ante las acciones de los jugadores. Esta información puede ayudar a los entrenadores a crear situaciones específicas de entrenamiento para mejorar el rendimiento de los porteros en las FD y aportar un conocimiento específico para el portero para preparar la estrategia adecuada en las FD en las situaciones de partido. Esta investigación ha permitido identificar que, ante la habilidad más utilizada por los jugadores, el dribling, el portero no responde utilizando las habilidades técnicas más efectivas. Esta información debe ser utilizada para corregir y mejorar las respuestas de los porteros.

En futuros estudios es importante aumentar la muestra de FD analizadas para poder llegar a un nivel de concreción más profundo gracias a poder analizar las diferentes categorías dentro de los disparos, arrastradas y driblings. De esta forma, se podrá aumentar el conocimiento de la vinculación entre portero y jugador y una mejor aplicación en la formación de estos.

\section{Agradecimientos}

Con el apoyo del Institut Nacional d'Educació Física de Catalunya (INEFC) de la Generalitat de Catalunya.

\section{Referencias}

American Psychological Association. (2002). Ethical Principles of Psychologists and Code of Conduct. American Psychologist, 57(12), 1060-1073. doi:10.1037/e305322003-001

Anguera, M.T., Blanco, A., Losada, J.L., \& Hernández-Mendo, A. (2000). La metodología observacional en el deporte: conceptos básicos. Lecturas de Educación Física y Deportes, Revista Digital, 24(5), 63-82.

Arboix-Alió, J., \& Aguilera-Castells, J. (2018). Influencia de marcar primero en hockey sobre patines. Cuadernos de Psicología del Deporte,

18(3), 220-231.

Baumann, F., Friehe, T., \& Wedow, M. (2011). General Ability and Specialization: Evidence From Penalty Kicks in Soccer. Journal of Sports Economics, 12(1), 81-105. doi:10.1177/1527002510371194

Bayer, C. (1987). Técnica del balonmano: la formación del jugador. Madrid: Hispano Europea.

Brazio, P. (2006). Estudo do processo ofensivo no Hóquei em Patins da Selecção de Portugal do Escalão Júnior (Tesi de Llicenciatura). Universidade de Porto, Porto.

Duque, G. (2004). Estrutura interna do jogo de Hóquei em Patins: Estudo exploratório das posses de bola no escalão de juvenis 
masculinos (Tesi de Llicenciatura). Universidade de Coimbra, Coimbra.

Espar, F. (2011). La táctica individual en el juego colectivo. In V. López \& J. Sargatal (Eds.), La táctica individual en los deportes de equipo

Ferreira, L. (2003). Estrutura interna do jogo de Hóquei em Patins: Estudo exploratório das posses de bola no escalão de seniores masculinos (Tesi de Llicenciatura). Universidade de Coimbra, Coimbra.

Folguera, C. (2000). Portería a cero. El entrenamiento del portero de hockey sobre patines. Barcelona: Carles Folguera.

González, P. (2008). El portero de hockey patines. Curso nacional de entrenadores de hockey patines (Material no publicado). Real Federación Española de Patinaje. ejercicios. Barcelona.

Hagemann, N. (2009). The Advantage of Being Left-handed in Interactive Sports. Attention, Perception \& Psychophysics, 71(7), 1641-1648. doi:10.3758/app.71.7.1641

Jimenez-Olmedo, J. M., Agulló, J. J. E., \& López, C. M. (2017). Análisis histórico de la efectividad de los lanzamientos a portería de balonmano masculino. Retos: nuevas tendencias en educación física, deporte y recreación, (32), 228-232.

Lasierra, G., \& Escudero, P. (1993). Observación y evaluación en los deportes de cooperación-oposición: en busca de aspectos distintivos. Apunts. Educació física i esports, (31), 86-105.

McMorris, T., \& Colenso, S. (1996). Anticipation of Professional Soccer Goalkeepers When Facing Right-and Left-Footed Penalty Kicks. Perceptual and Motor Skills, 82(3), 931-934. doi:10.2466/ pms.1996.82.3.931 (pp. 95-112). Girona: Diversitas 72.

González, P. (2011). El portero de hockey sobre patines. Cuaderno de

Parlebas, P. (2001). Juegos, deporte y sociedad. Léxico de praxiología motriz. Barcelona: Paidotribo.

Pauls, J. (2012) Táctica III: Alto rendimiento. Curso nacional de entrenadores de hockey patines. Barcelona (Material no publicado). Real Federación Española de Patinaje.

Pérez, M. D. C. I., Ordóñez, E. G., \& González, C. T. (2018). Gamerelated statistics in the Spanish Water Polo League: Differences Between Seasons. Retos: nuevas tendencias en educación física, deporte y recreación, (33), 228-232.

Pokrajac, B. (1980). Difference Between Initial Ball Velocites When Using a Sidearm Throw in Fieldball. Fizicka kultura, 34(4), 333337.

Rink Hockey Committee (2009). Reglas del juego. Lisboa:CERH.

Saavedra, J. M., Dorgeirsson, S., Kristjánsdóttir, H., Chang, M., \& Halldórsson, K. (2017). Handball Game-related Statistics in Men at Olympic Games (2004-2016): Differences and Discriminatory Power. Retos: nuevas tendencias en educación física, deporte y recreación, (32), 260-263.

Trabal, G. (2012). Portero III: Alto rendimiento. Curso Nivell 3 de entrenador de hockey patines 2012-13 (Material no publicado). Real Federación Española de Patinaje.

Trabal, G. (2016). Estudi etnogràfic del porter d'hoquei sobre patins: una vida entre paradoxes. Apunts. Educació Física i Esports, 126(4), 23-29. Doi:10.5672/apunts.2014-0983.es.(2016/4).126.02

Trabal, G. (2017). El porter d'hoquei patins en la falta directa a l'OK Liga. (Tesis doctoral inédita). Universitat de Barcelona, Barcelona.

Vaz, M., Ramos, N., Abrantes, J., Queirós de Melo, F., \& Conceiçao, F. (2011). Biomechanics of the Penalty Stroke in Roller Hockey. Revista Portuguesa de ciencias do desporto, 2(11), 129-132.

Zeier, U. (1987). O guarda-redes de Andebol. Cadernos 7 metros, 3, 2 48.

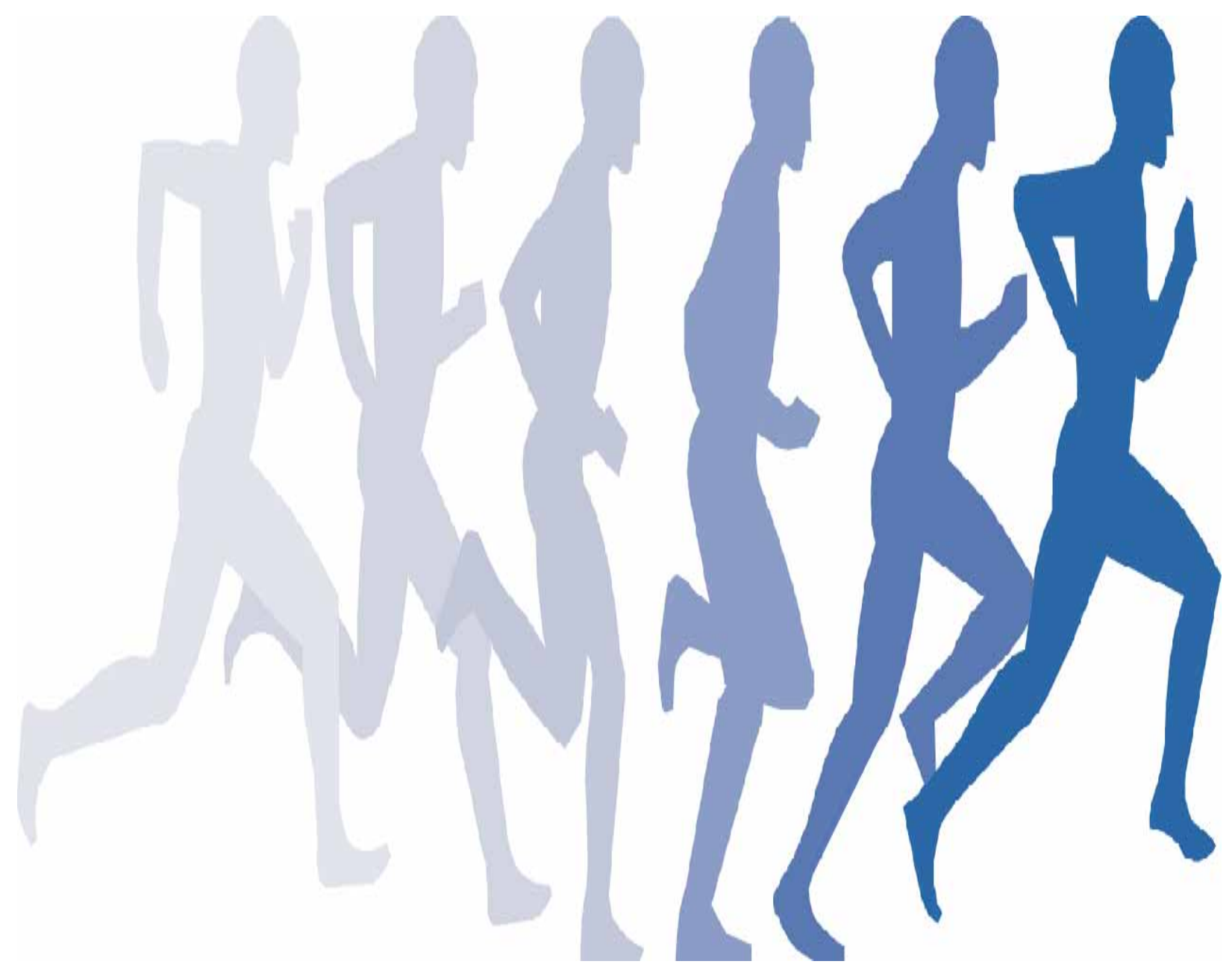

